\title{
Synchronous Interlocking of Discrete Forces: Strong Force Reconceptualised in a NLHV Solution
}

\author{
D. J. Pons ${ }^{1}$, A. D. Pons ${ }^{1}, \&$ A. J. Pons ${ }^{2}$ \\ ${ }^{1}$ Department of Mechanical Engineering, University of Canterbury, Christchurch, New Zealand \\ ${ }^{2}$ Rangiora New Life School, Rangiora, New Zealand \\ Correspondence: D. J. Pons, Department of Mechanical Engineering, University of Canterbury, Private Bag \\ 4800, Christchurch 8020, New Zealand. E-mail: dirk.pons@canterbury.ac.nz
}

\author{
Received: August 9, 2013 Accepted: August 23, 2013 Online Published: September 6, 2013 \\ doi:10.5539/apr.v5n5p107 URL: http://dx.doi.org/10.5539/apr.v5n5p107
}

\begin{abstract}
The conventional requirements for the strong force are that it is strongly attractive between nucleons whether neutral neutrons or positively charged protons; that it is repulsive at close range; that its effect drops off with range. However theories, such as quantum chromodynamics, based on this thinking have failed to explain nucleus structure ab initio starting from the strong force. We apply a systems design approach to this problem. We show that it is more efficient to conceptualise the interaction as interlocking effect, and develop a solution based on a specific non-local hidden-variable design called the Cordus conjecture. We propose that the strong force arises from particules synchronising their emission of discrete forces. This causes the participating particules to be interlocked: the interaction pulls or repels particules into co-location and then holds them there, hence the apparent attractive-repulsive nature of that force and its short range. Those discrete forces are renewed at the de Broglie frequency of the particule. The Cordus theory answers the question of how the strong force attracts the nucleons (nuclear force). We make several novel falsifiable predictions including that there are multiple types of synchronous interaction depending on the phase of the particules, hence cis- and trans-phasic bonding. We also predict that this force only applies to particules in coherent assembly. A useful side effect is that the theory also unifies the strong and electro-magneto-gravitation (EMG) forces, with the weak force having a separate causality. The synchronous interaction (strong force) is predicted to be intimately linked to coherence, with the EMG forces being the associated discoherent phenomenon. Thus we further predict that there is no need to overcome the electrostatic force in the nucleus, because it is already inoperative when the strong force operates. We suggest that 'strong' is an unnecessarily limiting way of thinking about this interaction, and that the 'synchronous' concept offers a more parsimonious solution with greater explanatory power for fundamental physics generally, and the potential to explain nuclear mechanics.
\end{abstract}

Keywords: unification, coherence, nucleon-nucleon interaction, residual strong force

\section{Introduction}

There is currently no way of showing how the strong force results in nuclear structures. This is problematic because it means that there is a conceptual gap between existing models for the strong force, e.g. quantum chromodynamics (QCD), and the elements of the periodic table and the nuclides. That there is a bonding relationship between protons and neutrons is evident, but the mechanism is unclear, and the existing theories struggle to model the simplest nuclei. Furthermore, other fundamentals, like the relationship of the electron to the nucleus, cannot currently be modelled from the perspective of the strong force, though the electron external orbital shapes are predicted by the Schrödinger wave equation.

This paper shows how the strong force can be reconceptualised, using a non-local hidden-variable (NLHV) design, specifically the Cordus theory (D. J. Pons, A. D. Pons, A. M. Pons, \& A. J. Pons, 2012). The previous work identified a proposed structure for the photon, and showed how this could solve wave-particle duality and entanglement. The present paper extends the concept to develop a theory that explains the mechanisms of the strong force. It is shown that this force can be explained by a synchronicity between the discrete forces of neighbouring particules. From this we predict that the synchronous interaction (strong force) has several variants. This is important, because it provides, as is shown in companion papers, a mechanism for the bonding of protons and neutrons, and hence explanations for nuclear assembly. 


\section{Existing Models of the Strong Force}

There are several levels to the strong force problem: (1) How do the quarks bond together within the nucleon? (2) How do the proton and neutrons bind within the hydrogen nucleus? (3) How do the nucleons form the elements and nuclides?

\section{Quantum chromodynamics}

The conventional approach is to start with the quark bonding situation, using quantum chromodynamics (QCD) theory (Gell-Mann, 1962; Nambu \& Han, 1974). QCD proposes that the strong force between quarks is created by gluons being exchanged between the quarks. In this model quarks have a property called colour charge (red, blue, green) which codes for the three distinct quantum states the particle can take (whereas particles more generally only have two states). Antiquarks take anticolours. Gluons are a type of virtual particle of which there are nine types. According to this theory, the quarks emit and receive gluon particles, which carry the colour charge. This exchange creates a force between the quarks, binding them together. This colour-force is believed to confine the quarks to the nucleon: it allows them some freedom within the nucleon but strongly prevents them making a wider separation, hence asymptotic freedom (Wilczek, 2005). Attempting to separate the quarks only causes a quark-antiquark pair to be produced instead. Gluons can interact between themselves, and this diminishes their effect at close range, thus the force is not infinitely strong at close range.

The force that holds the nucleons together is then explained as the residual effect of the gluons leaking outside the nucleons, hence residual strong force, or residual colour force. Thus the nuclear force is understood as arising from the exchange of pi mesons (pions) between nucleons, in the Yukawa interaction (Yukawa, 1947). Pions $\left(\pi^{0}, \pi^{+}, \pi^{-}\right)$are combinations of up and down quarks, where one of each pair is the antimatter type. The explanation for the bonding between neutrons is that these point structures are polarised such that a residual element of the strong force binds them together. The mechanism for the repulsive nature of the nuclear force is the gluons interacting with each other.

The theory of QCD is generally considered successful. It offers a conceptual model (gluons and colour-force), and an associated mathematical representation. QCD provides predictions that are consistent with outcomes measured at colliders, e.g. three-jet events. It is an important part of the standard model of physics, wherein it provides the framework for modelling the inner structures of the nucleons.

However QCD is not without problems. The strong force is explained in terms of a colour-force, which thus also needs an explanation. This adds to the overall problem, because the fundamental mechanisms of force are unknown. How does exchange of particles cause force to arise? Adding more forces, colour in this case, may be useful for explaining quark behaviour, but is not parsimonious for the wider problem, and makes it harder to achieve a unified explanation for force. Also, attempts to extend QCD's mathematical formulation have not increased its explanatory power. More problematic still is that QCD only addresses one of the fundamental questions: how the quarks bond together. It is not useful in answering the second question, how the nucleons join together. QCD is vague about the larger scale bonding of protons and neutrons within the nucleus, and the concept of polarised points is paradoxical. It is quite unable to explain the stability or instability of specific nuclides. Therefore, while QCD might be a useful theory for quark bonding, it has not yet demonstrated any relevance to the wider problem of nuclear structures.

\section{Nuclear models}

At the nuclear level there are a number of other theories: shell model (Ivanenko, 1985), liquid-drop model (Gamow, 1930), and semi-empirical mass formula (SEMF) (Weizsäcker, 1935). These theories, particularly the SEMF, seek to model the BE characteristics. None of these theories makes any use of the strong force per se, and certainly not of the gluon theory. The SEMF is able to model binding energy reasonably well, but there is a further problem in that binding energy is an inaccurate predictor of nuclide stability. None of these theories are able to differentiate stable from unstable nuclides, or explain anything but the most general trends in the table of nuclides. Consequently, while there logically should be a theoretical progression from the strong force to nuclear structure, this simply is not the case.

\section{Nuclear binding energy}

There is a body of empirical evidence in the form of binding energy (BE), proton separation energy, and neutron separation energy, for each nuclide. It is commonly accepted that the nuclear binding energy holds the nucleus together, where that energy is created from the mass deficit between the masses of the individual components compared to the mass of the assembly. But that is of itself not an explanation, because it does not explain the mechanism whereby the mass deficit is converted into binding force. Nor does it explain why that force, once 
created and sufficient to overcome the electrostatic repulsion, does not simply crush the nucleus into a singularity. A further problem is that binding energy is unable to discriminate reliably between stable and unstable nuclides, and for this reason alone cannot be the main determinant of stability. Therefore attempts, like the SEMF, to model binding energy, are unlikely to yield a successful theory of the nuclides.

\section{Gaps in the body of knowledge}

The predicament is that the current models of the strong force are unable to predict nuclear structure. This is problematic, given that the strong force was originally conceived as a method of binding the protons and neutrons together in the nucleus. Given the fundamental theoretical discrepancy in this area, there must be deeper relationships of causality than captured in any of the existing theories, i.e. new mechanics must exist.

\section{Purpose and Approach}

\section{Purpose}

The purpose of this paper is to prospect for a different foundational concept for the strong nuclear force, one with the capability to coherently explain the nuclides. We do this in several parts. First, and the subject of the present paper, we develop a novel theory for the strong force. This theory replicates all the attributes of that force: its weak effect at long range, its strength at short range, and its repulsive effect at the inner range. This new theory also achieves a conceptual unification of the electrostatic, magnetic, gravitational, and strong forces. Compared to QCD it is simpler, more parsimonious in its use of variables, and explains more. In the second and subsequent parts, not covered here, we apply this strong force theory to the bonding of protons and neutrons, and the structure of the nuclides.

\section{Approach}

To achieve this objective, we start by recognising that the problem is fundamentally a conceptual one, as opposed to a mathematical one, and we select our methodology accordingly. We use a systems engineering design method. This involves taking the functional requirements (observed behaviour of the strong force) and inferring the requisite attributes (internal and external mechanics of matter particules).

\section{Functional requirements: The conventional specification for the strong force}

The required attributes of the strong force are conventionally understood as follows. These are inferred from the behaviour of the protons and neutrons in the nucleus.

1) The nuclear force is required to overcome the electrostatic force of repulsion between protons. It needs to be 'strong' as the electrostatic force becomes stronger as the separation decreases.

2) Also, the strong force is required to exert its attraction between nucleons, whether they are neutral or charged. So it cannot be a charge-effect in the usual sense of charge.

3) And while the nuclear force needs to be stronger than the electrostatic force, it needs to weaken at distance so that it does not interfere with that other force.

4) Moreover, the nuclear force needs to turn into a repulsive force if the nucleons come too close, or it would crush the nucleus.

These expectations create a mental model of what the force should be like, and even the name, 'strong' is a consequence of the construct.

\section{Questioning the premises}

Since we are undertaking conceptual work, we make a note to question these premises and their logical structure. We have to be open to the possibility that they frame the problem inefficiently, and perhaps even erroneously.

We do not disagree with the output behaviour of the system, i.e. that protons repel each other, or that protons and neutrons are bonded in the nucleus. However we do question the way these behaviours are conventionally interpreted. For a start, we question the assumption that the strong force has three levels of operation: weak Strong force at long range $>$ strongly attractive Strong force at middle range $>$ repulsive Strong force at close range. Is it really necessary to have a force that changes its characteristics? The conventional approach first needs to have a mechanism for a 'strong' force. Then it needs to have a mechanism to dilute that force at large range. Then a third mechanism is required to change the force into a repulsive one. Is there no more efficient way?

Therein lies the problem, because any solution that seeks to explain the conventional Specification is inefficient, from a design perspective. More so when electrostatic repulsion is added to the mix. Inefficient, in that it 
requires diverse mechanisms and many variables. There is a possibility that a more efficient design might exist, one with fewer mechanisms and greater explanatory power.

\section{Refining concepts by evaluating fitness}

The design process is then to take the existing functional requirements, and any new candidate concepts, and check whether by extending those concepts it is possible to provide a solution.

In this case the candidate starting concept is the Cordus conjecture. This is a NLHV solution that has been specifically designed to explain entanglement, wave-particle duality, and optical laws for reflection and refraction (D. J. Pons, A. D. Pons, A. M. Pons, \& A. J. Pons, 2012). It predicts that photons and matter have a specific internal structure. This structure is called a 'particule', to distinguish it from the point-particle more commonly used in physics. While such a NLHV solution may seem precluded by the Bell-family of inequalities (Bell, 1964; Leggett, 2003) there is reason to believe those constraints do not exclude absolutely all possibilities (De Zela, 2008; Groblacher et al., 2007; Laudisa, 2008). This Cordus theory also predicts the existence of discrete forces and hence the electro-magneto-gravitational (EMG) forces and fields. It also proposes the internal structure of the electron and anti-electron (positron) in terms of the Cordus framework, and identifies that the matter-antimatter species are differentiated by the hand of the discrete forces, specifically the emission sequence. Starting from this prior work, we now extend the concept to create a theory for how the strong interaction works.

In general the design process results in many variants, and the more promising have to be identified, tested against the functional requirements, and further developed as necessary. Design is always an integrated problem-solving endeavour, which in this case means that the solution has to be reviewed until it is also logically consistent with -or changes- all the other prior lemmas of the Cordus theory. This is necessary for building a coherent solution, as opposed to a solution, like QCD, that only works in the specific area under examination. The results of this design process are shown below.

\section{Results}

We start by summarising the Cordus theory, since it is novel and not widely known. We then explore how its discrete force structures provide a solution to the strong force problem. We then use that to predict how the strong force operates between protons and neutrons in this theory.

\subsection{Cordus Theory for Matter}

The Cordus conjecture postulates that all particles are one dimensional structures of finite length, and from their two ends emit three-dimensional discrete forces that travel down flux lines. This is called a particule.

\section{Inner structure of the Cordus particule}

The basic idea is that every particule has two reactive ends, which are a small finite distance apart (span), and each behave like a particle in their interaction with the external environment (D. J. Pons, A. D. Pons, \& A. J. Pons, 2012). A fibril joins the reactive ends and is a persistent and dynamic structure but does not interact with matter. It provides instantaneous connectivity and synchronicity between the two reactive ends. Hence it is a non-local solution: the particule is affected by more than the fields at its nominal centre point. Each reactive end of the particule is energised in turn at the frequency of that particule (which is dependent on its energy). The reactive ends are energised together for the photon, and in turn for matter particules. The frequency corresponds to the de Broglie frequency. The span of the particule shortens as the frequency increases, i.e. greater internal energy is associated with faster re-energisation sequence, hence also faster emission of discrete forces (see next section) and thus greater mass.

\section{External structure: Cordus discrete field structures}

When the reactive end is energised it emits discrete forces in up to three orthogonal directions. The quantity and direction of these are characteristic of the type of particule (photon, electron, proton, etc.), and the differences in these signatures is what differentiates the particules from each other. Although for convenience we use the term discrete force for these pulses, the Cordus theory requires them to have specific attributes that are better described as latent discrete prescribed displacements. This is because a second particule that subsequently receives one is prescribed to energise its reactive end in a location that is slightly displaced from where it would otherwise position itself. Thus in the Cordus theory, that which we perceive as force is fundamentally the effect of many discrete prescribed displacements acting on the particules.

These discrete forces are connected in a flux line that is emitted into the external environment. (Note 1) (In the Cordus theory this is called a hyperfine-fibril, or hyff). Each reactive end of the particule emits three such 
orthogonal hyff, at least in the near-field. The exception is the photon, which only emits radially. These directions are relative to the orientation of the span, and the velocity of the particule, and termed hyperfine-fibril emission directions (HEDs). The axes are named [r] radial outwards co-linear with the span, [a] and [t] perpendicular to the span and to each other. These are so-named for consistency with previous nomenclature for the photon (D. J. Pons, A. D. Pons, A. M. Pons, \& A. J. Pons, 2012), but when applied to massy particules do not necessarily imply motion. It is proposed that the quarks and other leptons follow the same pattern, though in the case of the quarks not all the hyff emission directions $[\mathrm{r}, \mathrm{a}, \mathrm{t}]$ are filled (hence their fractional charge). These general principles are shown in Figure 1, and their application to the photon in Figure 2.

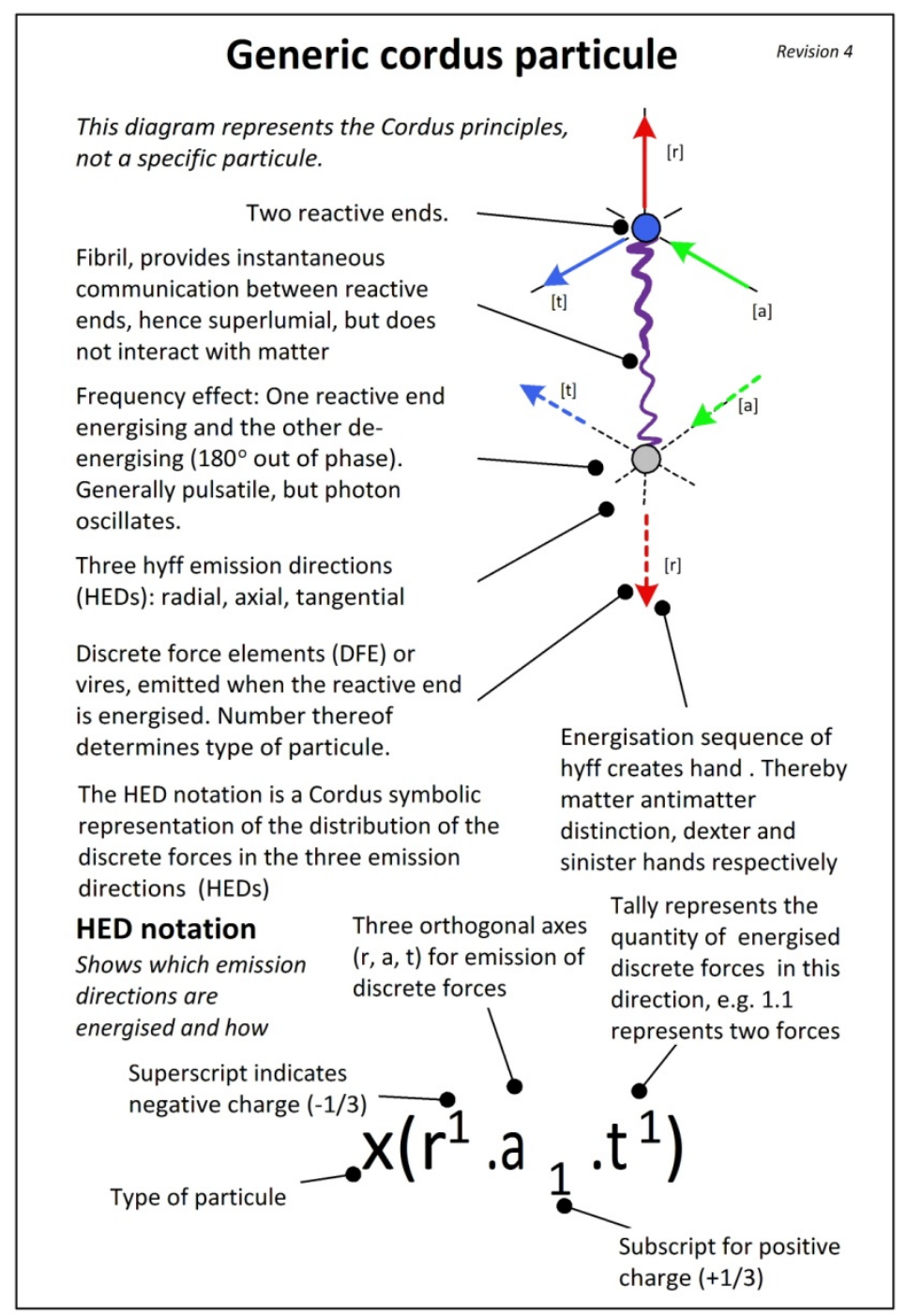

Figure 1. The Cordus theory proposes that particules have an internal structure and emit a signature of discrete external forces. This diagram shows the generic principles 


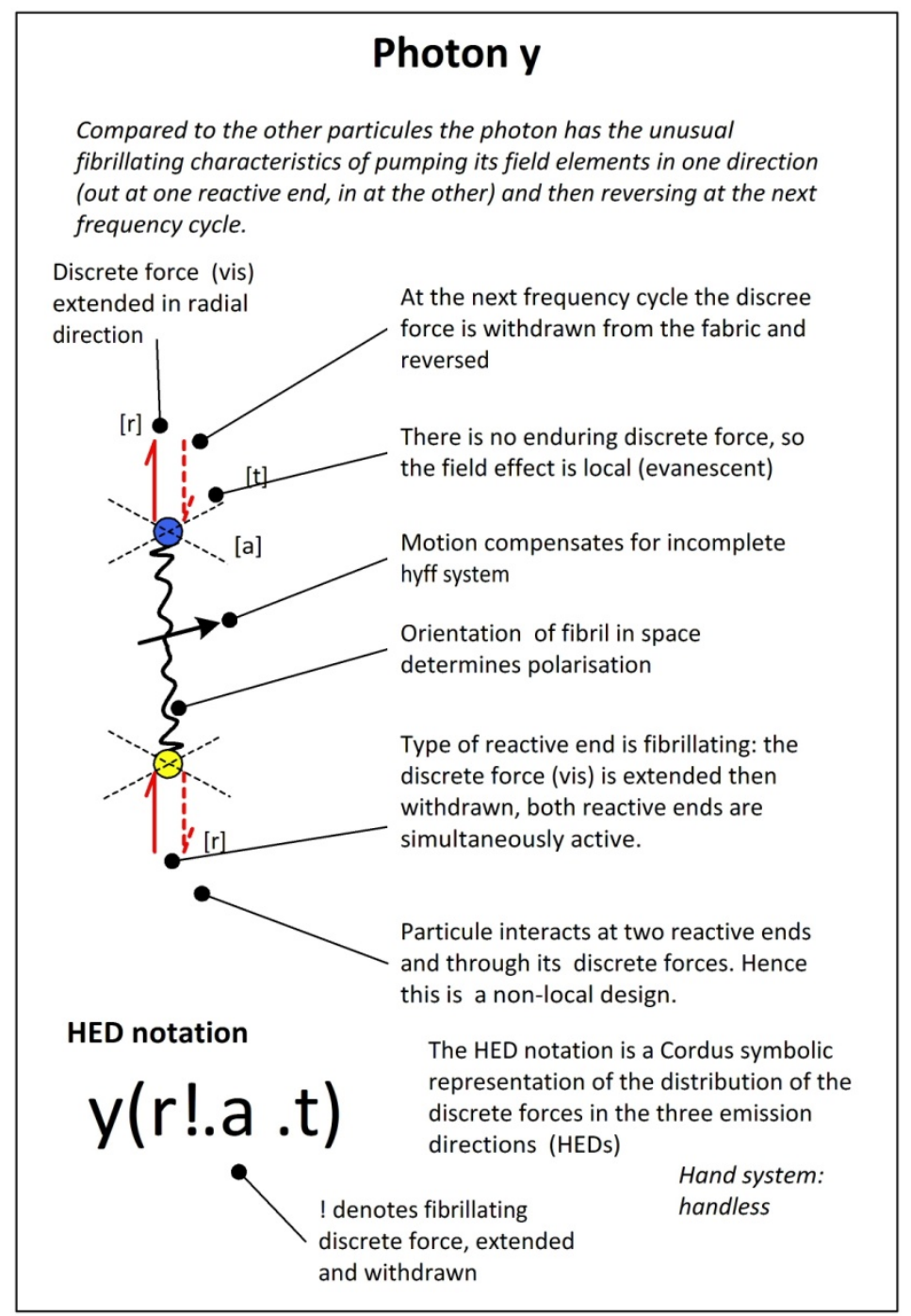

Figure 2. The Cordus theory for the photon structures

The aggregation of discrete forces from multiple particules creates the EMG fields, which are thus discrete. The combined emission discrete forces makes up a 3-D composite structure. The direct lineal effect of the discrete force provides the electrostatic interaction, the bending of the hyff flux line provides magnetism, the torsion provides gravitation interaction, and the synchronicity between discrete force elements of neighbouring particules provides the strong force. These are all carried simultaneously by the composite discrete force element as it propagates outwards on the hyff flux.

Assembled massy particules compete spatially for emission directions, and may synchronise their emissions to access those spaces. Thus there is mutual negotiation in the near-field between interacting particules, based on shared geometric timing constraints. These particules interact by negotiating complementary HEDs and synchronising the emission frequencies of their discrete force elements. This synchronicity is proposed as the mechanism for the strong force, as shown here, and for coherent assemblies. The same mechanism, acting through coherent assemblies of electrons, explains molecular bonding. Thus the Cordus theory provides force unification by providing a theory for electro-magnetic-gravitational-synchronous (EMGS) interactions as consequences of lineal, bending, torsion, and synchronicity effects respectively. The discrete force element is a 3 -D composite structure, with a hand defined by the energisation sequence between the axes. This hand provides the matter/anti-matter species differentiation (D. J. Pons, A. D. Pons, \& A. J. Pons, 2013).

In this theory electric charge is carried at $1 / 3$ charge per discrete force, with the sign of the charge being determined by the direction of the discrete force element. So the number and nature of energised HEDs determines the overall electric charge of the particule. For example, the electron is proposed to have three 
discrete field elements, see Figure 3. Neutral structures are accommodated, but incompletely filled HEDs are proposed as the reason for instability and decay (D. J. Pons, 2011).

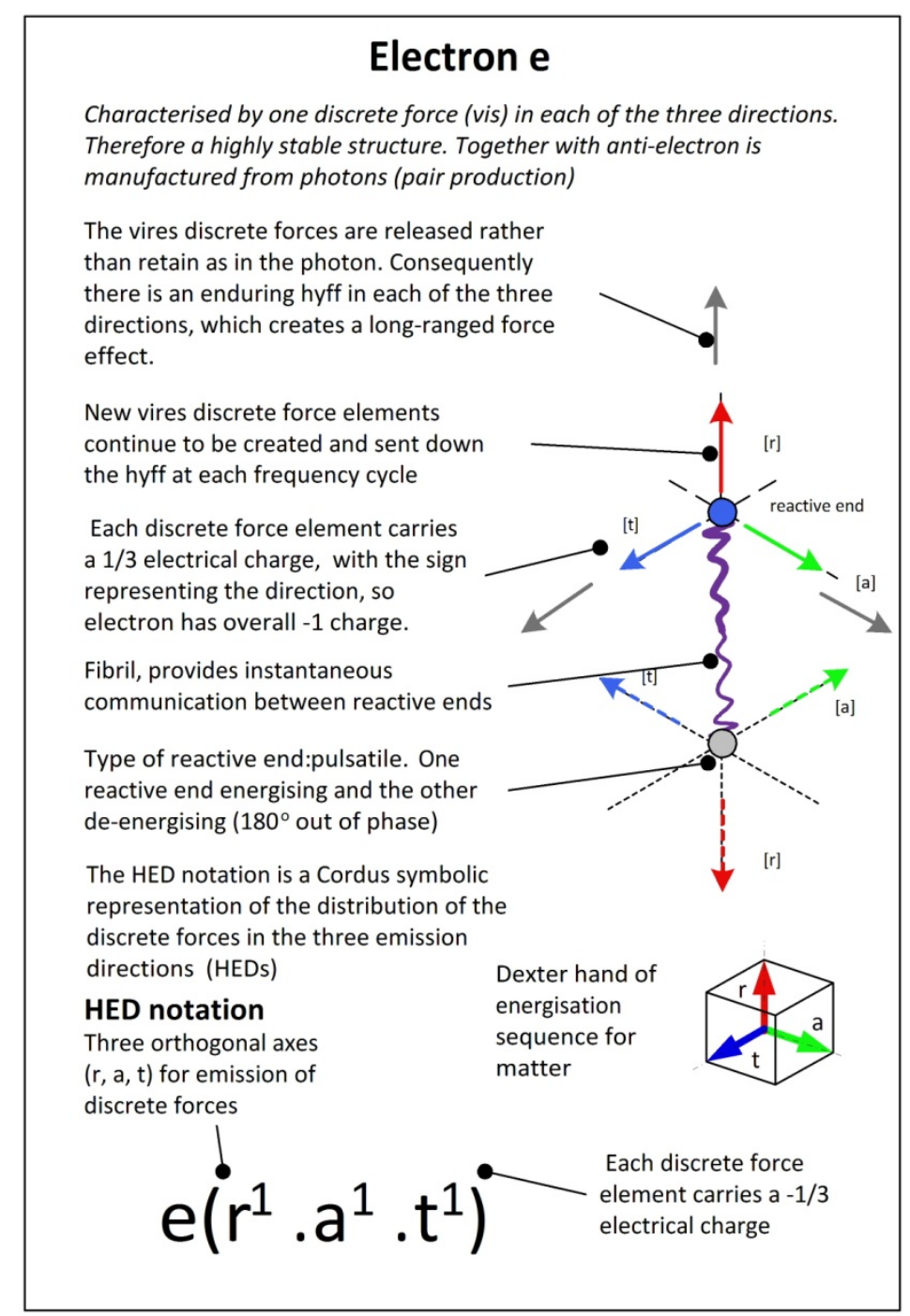

Figure 3. Cordus theory of the electron. It is proposed that the particule has three orthogonal discrete forces, energised in turn at each reactive end

The HED notation is a shorthand symbolic representation of the HED arrangements for this particule, and includes the three axes and the number and direction of discrete forces in each (superscripts are negative charge, subscripts positive charge). For antimatter the axes and field system takes the other hand. The proposed structure for the proton is shown in Figure 4 and for the neutron in Figure 5. This is derived from consideration of the known quark content and their charges. (Note 2) 


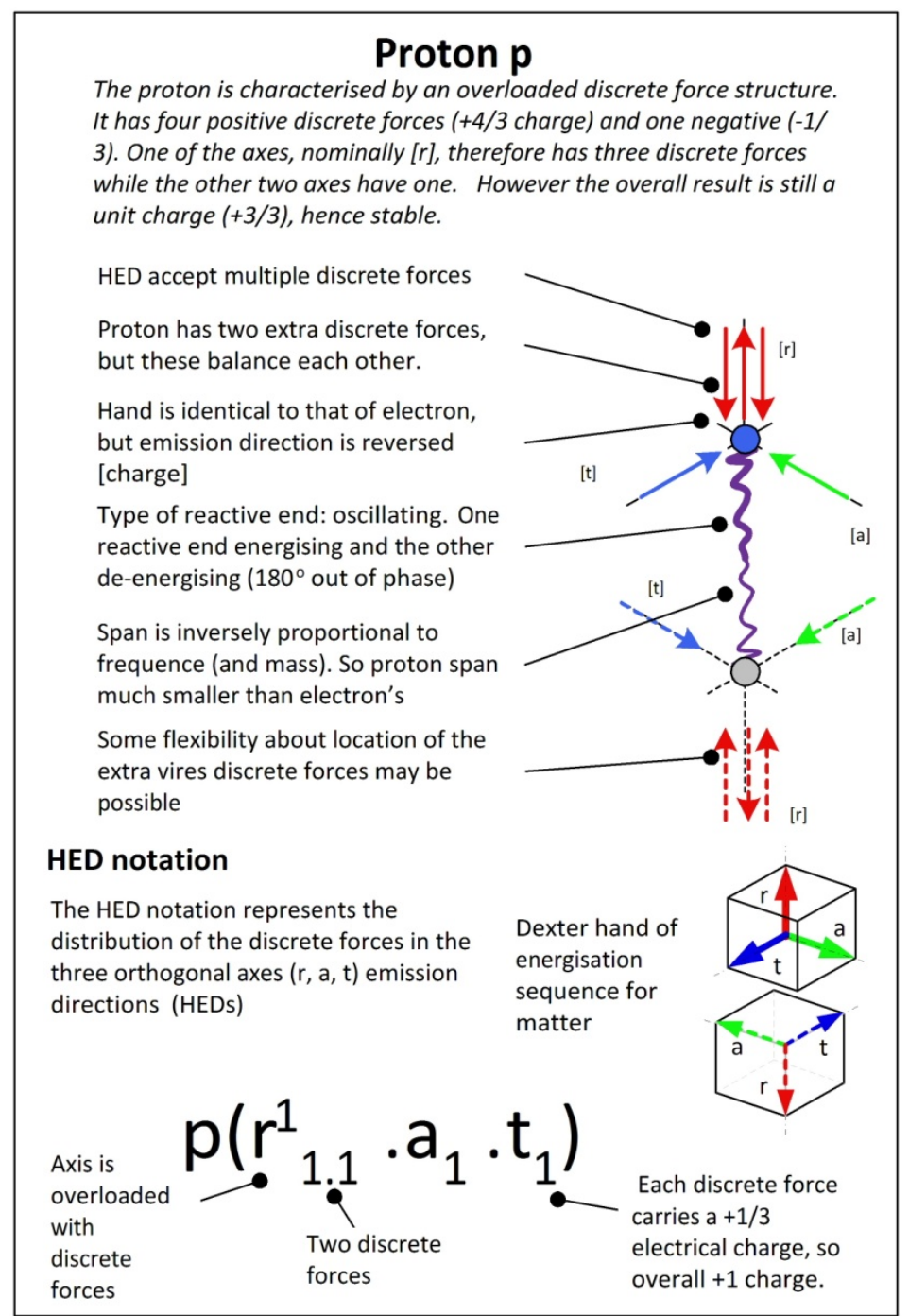

Figure 4. Cordus theory for the proton. The distinguishing feature of this particule is the overloaded discrete forces. The higher mass of the particule, compared to say the electron, is proposed to arise from the higher frequency of re-energisation for this particule, in turn driven by internal fibril dynamics not apparent here

The Cordus theory may look strange compared to the zero-dimensional point construct that dominates our mental models of physics. The idea is radical, and certainly is unorthodox. Nonetheless it offers explanations for many fundamental phenomena (D. J. Pons, A. D. Pons, \& A. J. Pons, 2013; D. J. Pons, A. D. Pons, \& A. J. Pons, 2011; D. J. Pons, A. D. Pons, A. M. Pons, \& A. J. Pons, 2012), and thus has high conceptual fitness. It offers new insights and interesting new possibilities. We explore one of these regarding the strong force. 


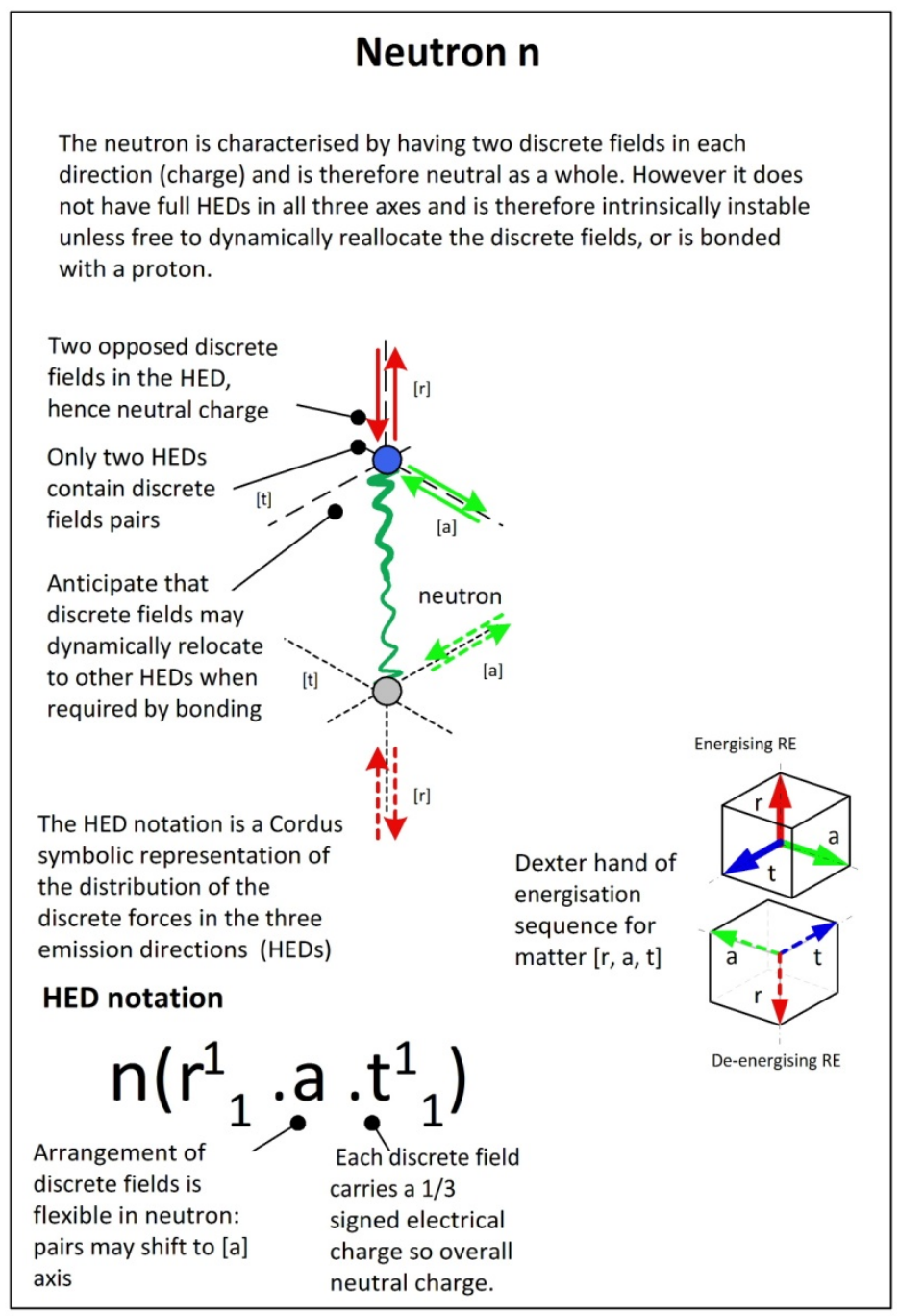

Figure 5. Cordus theory for the neutron. The distinguishing feature of this particule compared to the proton is the balanced number of inwards and outwards discrete forces, hence neutral charge

\subsection{Theory of the Strong Nuclear Interaction}

We now introduce a major point of departure relative to the conventional specification for the strong force. That specification requires the force to change its effect at different ranges, from being weak, to strongly attractive, to repulsive. Instead, we suggest that all these behaviours can be explained in a totally different way:

We propose that the strong nuclear interaction arises from the synchronisation of discrete forces between particules, and is thus an assembly interlock effect.

This simple proposition has a number of far-reaching implications. We expand on the idea in the following proposals:

\section{(1) Strong force arises from the synchronisation of discrete forces between particules}

According to the Cordus interpretation the strong interaction is the synchronous interlocking between discrete forces (discrete field elements), the action of which tends to pull or repel particules into co-location and then hold them there. This force only applies to particules in coherent assembly. Coherence, including the causes and limited thereof, is discussed elsewhere.

Thus the strong force arises from the need for particules to share hyff emission directions. This applies when they come sufficiently close to each other. Particules can also completely co-locate, i.e. geometric superposition of reactive ends from different particules. (However, this is not the same as the temporal superposition of 
quantum mechanics, which the Cordus theory rejects.) Since the discrete force pulses are discrete, being emitted at a frequency, it is necessary for particules to synchronise their emissions in location, frequency, and phase, if they wish to remain co-located. Thus the strong force is a stabilising effect due to synchronous hyff emission directions (SHEDs).

\section{(2) Discrete forces are the common underlying mechanism for all forces (interactions)}

The Cordus theory leads us to propose that that there is a single fundamental mechanism, the discrete force, for the strong force and the electron-magnetic-gravitational (EMG) forces. The four hyff variables are synchronicity, strength, bending, and torsion (hand), respectively. Thus it is proposed that all interactions between particules are mediated by discrete forces.

The proposed mechanism for force and hence field interactions at a distance is as follows: the basal particule A emits discrete forces which propagate outwards $>$ one of these forces is intercepted by remote particule $\mathrm{A}>$ the interception involves the discrete force from $\mathrm{A}$ interfering with emissions at $\mathrm{B}>$ this interference causes the reactive end of $\mathrm{B}$ to energise in a slightly different location (closer, further, or laterally, as the case may be) than otherwise $>$ this displacement is what we perceive as a force acting on $\mathrm{B}$, since we have to exert a reactive constraint to prevent the motion, hence Newton's third law $>$ the discrete force from A is not consumed in the interaction but continues its outward propagation, and is available to react with more remote particules $>$ A continues to emit discrete forces, and $\mathrm{B}$ continues to receive them $>$ since the frequency is high, the overall effect is that of a smooth field or force. To summarise this aetiology, the Cordus theory proposes that force arises by discrete forces constraining the position of re-energisation of the reactive end of the recipient particule, i.e. force is fundamentally a prescribed displacement effect.

The synchronous interaction (strong force) is slightly different in that it is not a ranged effect, but rather a frequency interlock between co-located reactive ends, which causes them to fix their positions, as opposed to move as in the EMG forces. However in both the synchronous and EMG cases the fundamental effect is a constraint on displacement. (Note 3)

Hence the attractive-repulsive nature of the strong force is readily accommodated: the particules that attempt to decouple their reactive ends will experience very strong constraints to bring them back together. The force is also predicted to have preferential directionally alignment. Indeed, if one accepts our assertion that the synchronous interaction only applies to coherent assemblies of matter (see next section) then one can interpret the spin characteristics of superfluids and Bose-Einstein condensates as a macroscopic manifestation of the synchronous interaction between electrons of different atoms.

\section{(3) Strong force is proposed to be a different category of force to EMG and exclusive to coherent assemblies}

The Cordus theory leads us to propose that the strong force is a fundamentally different type of force category to the macroscopic electro-magneto-gravitational forces. We propose that the differentiating factor is the synchronicity and hence coherence (or lack thereof) of the participating particules.

- $\quad$ For the strong force, the interacting particules necessarily have synchronous frequencies, i.e. emit their discrete forces at one common frequency, and interact synchronously. Thus these particules are in coherence with each other.

- For the EMG forces there need be no synchronicity. The Cordus theory is that the electrostatic, magnetic, and gravitational forces are macroscopic forces. They are explained as arising from the disjointed rain of discrete forces on the recipient particule. Thus the fields are all discrete, but their macroscopic effect is practically smooth and continuous.

The EMG forces therefore apply to both coherent and discoherent bodies, but the synchronous strong force only to coherent bodies.

Thus central to this theory is a proposed new concept of coherence and discoherence, for which we have elsewhere provided a more detailed theory and explanation (D. J. Pons, 2012). The Cordus theory suggests that the strong force and coherence are both manifestations of a single deeper mechanics: the coordinated access to synchronised emission directions of the flux tubes (hyff).

\section{(4) Strong force gives rise to specific bonding structures}

We propose that the strong force produces a variety of specific structural assemblies between particules. This only applies in coherent situations, see above. The differentiating factor is proposed to be the phase offset of the frequency of the particules (spin). Note the assumption that coherent particules already have a common 
frequency. There are two discrete states that particularly interest us: the particules may be in or out of phase with each other, which we term cis- and trans-phasic behaviour.

\section{(5) Annihilation is another manifestation of the strong force}

In the Cordus theory the differentiating factor between matter and antimatter is the hand of the reactive end. The mechanisms for annihilation are also anticipated by the Cordus conjecture. Here we simply propose that the interaction at annihilation is another sub-type of the strong interaction. We propose that the synchronisation of discrete forces from same-handed particules results in an additive effect and hence bonding, whereas the interaction of dissimilar handed species results in cancellation of discrete forces and hence annihilation.

\section{(6) Singularity avoidance}

This theory also explains why the electron that is bonded to a proton is not merged into the proton, something which is problematic to conventional models. The electron has two reactive ends, and only one can be co-located with any one reactive end of the proton. So the electron always has a span, and therefore an identity. In this way the Cordus theory also avoids the singularity problems that trouble the 0-D point model.

\section{(7) Energy equity mechanism}

A general characteristic of all the strong force interactions in the Cordus theory is that participating particules are coupled together. The need to preserve the same re-energising locations means that the energy systems are joined. They can (and must) redistribute excess incoming energy between them. (Note 4) This transmission is predicted to be within a frequency cycle, if not instantaneous, and hence the overall effect can be superluminal if the assembly can be contrived to exist coherently at a macroscopic scale. Single particules, e.g. photons, are always internally coherent, hence capable of exhibiting this instantaneous coordination between reactive ends that are some distance apart, and we propose this as the explanation for entanglement (D. J. Pons, A. D. Pons, A. M. Pons, \& A. J. Pons, 2012). Another effect is photon emission. If an atomic assembly cannot contain all the energy it receives, then either it must disassemble, or emit the surplus as a photon.

This also explains several other effects. The first is mass deficit. Here the Cordus explanation is that the assembly of the subcomponents (quarks into nucleons, or nucleons into nucleus), requires for synchronicity that they have a common frequency. This may be different to the native frequencies of those subcomponents. Consequently the subcomponents may have a different frequency in the assembly to that which they adopt when isolated. In the Cordus theory, as in conventional physics, frequency corresponds to mass, and hence the mass of subcomponents may differ from that of the assembly.

The Cordus theory also offers a mechanism for mass: that the number of handed discrete forces emitted, and the frequency of emission, determine the mass of the particule. This is because the frequency of a particule is determined by its internal energy, as per the $\mathrm{E}=\mathrm{hf}$ relationship. Hence higher energy in a particle results in a higher frequency, thus quicker emission of discrete forces, and ultimately a greater mass effect. The mass effect is measured either by its gravitational force on a remote test object, or by its response to an accelerating force. We do not have space here to fully explore gravitational-inertial mass equivalence, but merely point out that in both cases the measurement of mass involves forces, and that the Cordus theory explains force as a prescribed constraint on the re-energisation position of the recipient reactive end. Note also that the Cordus theory proposes that the span of the particule is inversely proportional to its frequency (hence also to its energy or mass). This explains why smaller particules have greater mass (or energy). The smaller the subatomic particle needs to be (for assembly) reasons, the shorter its span must be, and hence the greater its frequency needs to be - which takes energy and is evident in its mass. This also explains some otherwise ontologically problematic observations regarding the size of the proton.

Existing methods of attempting to measure the 'diameter' of the proton involving measuring its interaction with electrons, either in bonding situations or impact-scattering. From a Cordus perspective these experiments are measuring the average interaction geometry of the electron and proton, not a physical diameter. It is natural to call this the 'diameter' of the proton, but that really is only an interpretation based on the a priori assumption that a particle should be a sphere of charge. The Cordus theory suggests that the measurement is dependent on the probing particle. This is consistent with the observation that the diameter of the proton is measured to be smaller when the muon is used as the probing particle. The Cordus explanation is that the proton and electron/muon bond via the synchronous interaction, which by definition requires that they negotiate and adjust their frequencies to a common or harmonic value. In turn the span is inversely proportional to frequency, so the frequency adjustment necessarily also changes the span. Thus the effective interaction geometry of the proton depends on the frequencies of the participants. If the electron is replaced with a heavier muon, then the muon 
will negotiate a higher synchronous frequency with the proton, than would the electron. This means that the proton will also have a higher frequency than otherwise, hence a shorter span and greater frequency (D. J. Pons, A. D. Pons, A. M. Pons, \& A. J. Pons, 2011a). This is exactly what is observed: the proton in muonic hydrogen is measured to have a smaller 'diameter' and greater mass than in electronic hydrogen. The underlying mechanism in all these cases is proposed to be the tight coupling of frequencies in the Cordus synchronous interaction.

\section{(8) Spin}

In the Cordus theory spin refers to the orientation and frequency state of the central fibril of a single particule. The necessary parameters are two angles describing the orientation of the span $\varphi_{1}$ and $\varphi_{2}$ relative to the coordinate system of choice - these are polarisation angles-, a third angle $\varphi_{3}$ denoting the orientation of the [a] axis, the phase angle $\theta$ for the energisation sequence, and the frequency $\omega$ of the particule. Multiple particules that are discoherent have their own independent parameters for all these variables. These particules interact via the EMG forces. For two (or more) particules in a coherent relationship, then the synchronous interaction applies. This fixes the frequency of the particule $\omega$ to a common or harmonic value. The interaction also makes the three orientation angles $\varphi_{1}, \varphi_{2}$ and $\varphi_{3}$ into local constants, so they are no longer variables. The only remaining variable is the phase angle $\theta$, which in the coherent case is either cis- or trans-phasic. The Cordus theory identifies cis-phasic $\theta=0$, and trans-phasic $\theta=\pi$. Thus the Cordus phase angle $\theta$ corresponds to the quantum mechanics spin angular momentum $\mathrm{n} / 2$ (which typically takes the value of $1 / 2$ or 1 ). In contrast $\mathrm{QM}$ identifies the two angles of polarisation $\varphi_{1}, \varphi_{2}$, spin $\mathrm{n} / 2$, and frequency $\omega$, but treats them as intrinsic variables without physical interpretation. Thus the Cordus theory is consistent with the empirical observation that coherent particules have only two choices in their interaction spin, and provides a natural explanation for this.

\subsection{Application to Proton-Neutron Bonding}

The synchronous interaction proposed here readily explains the bonding of a single proton and neutron. Specifically it is proposed that the proton and neutron bond in a cis-phasic manner, by synchronising the emission of their discrete forces, as shown in Figure 6. Note that the assembly results in each of the three axes having equal quantities of signed discrete forces. We propose this balance is the reason for stability of the assembly. 


\section{Approximation: Approach of two particules}

Neutron and proton have different arrangements of their discrete forces, both the arrangement across the three axes, and the direction (charge)

\section{Coordinate} system

Dexter hand of energisation sequence for matter

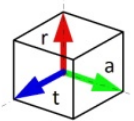

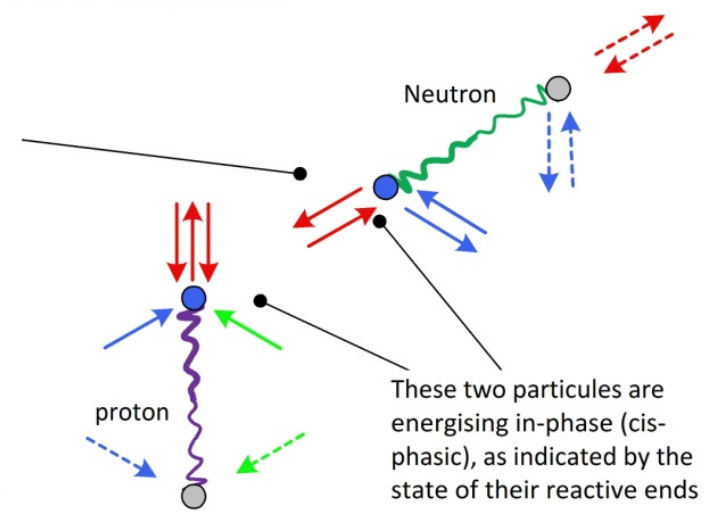

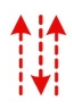
phasic), as indicated by the

(1) 个is

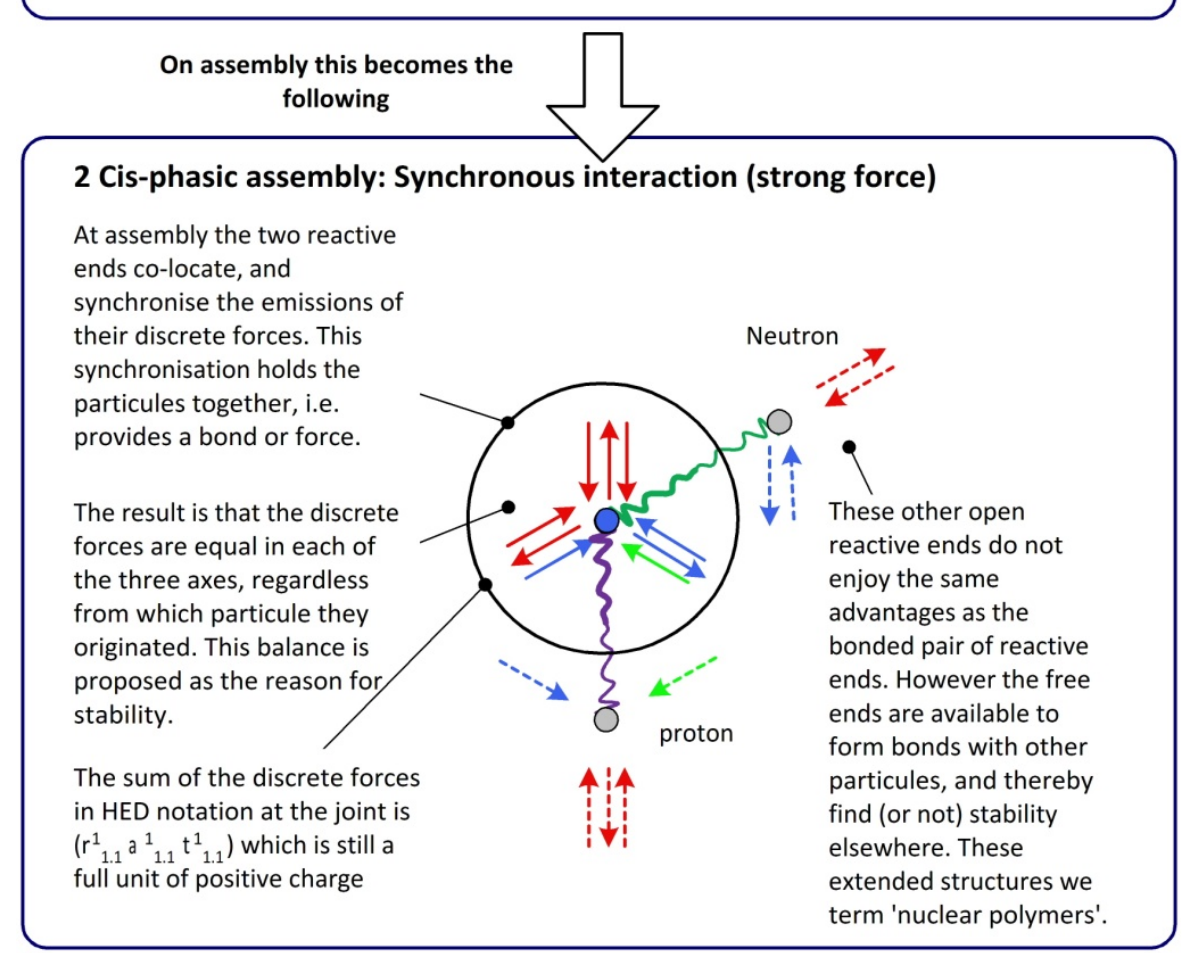

Figure 6. Cordus theory for synchronous interaction (strong force) bonding the proton and neutron

This illustrates that the Cordus synchronous interaction has the power to explain the usual features of the strong force, and offer a solution to the problem of now the nuclear strong force operates to assemble nuclear structures.

\subsection{Categorisation of the Strong Force}

Taken together, these proposals allow us to construct a categorisation diagram of the interactions, summarised in Figure 7. The categorisation is primarily regarding synchronous vs. asynchronous interactions. 


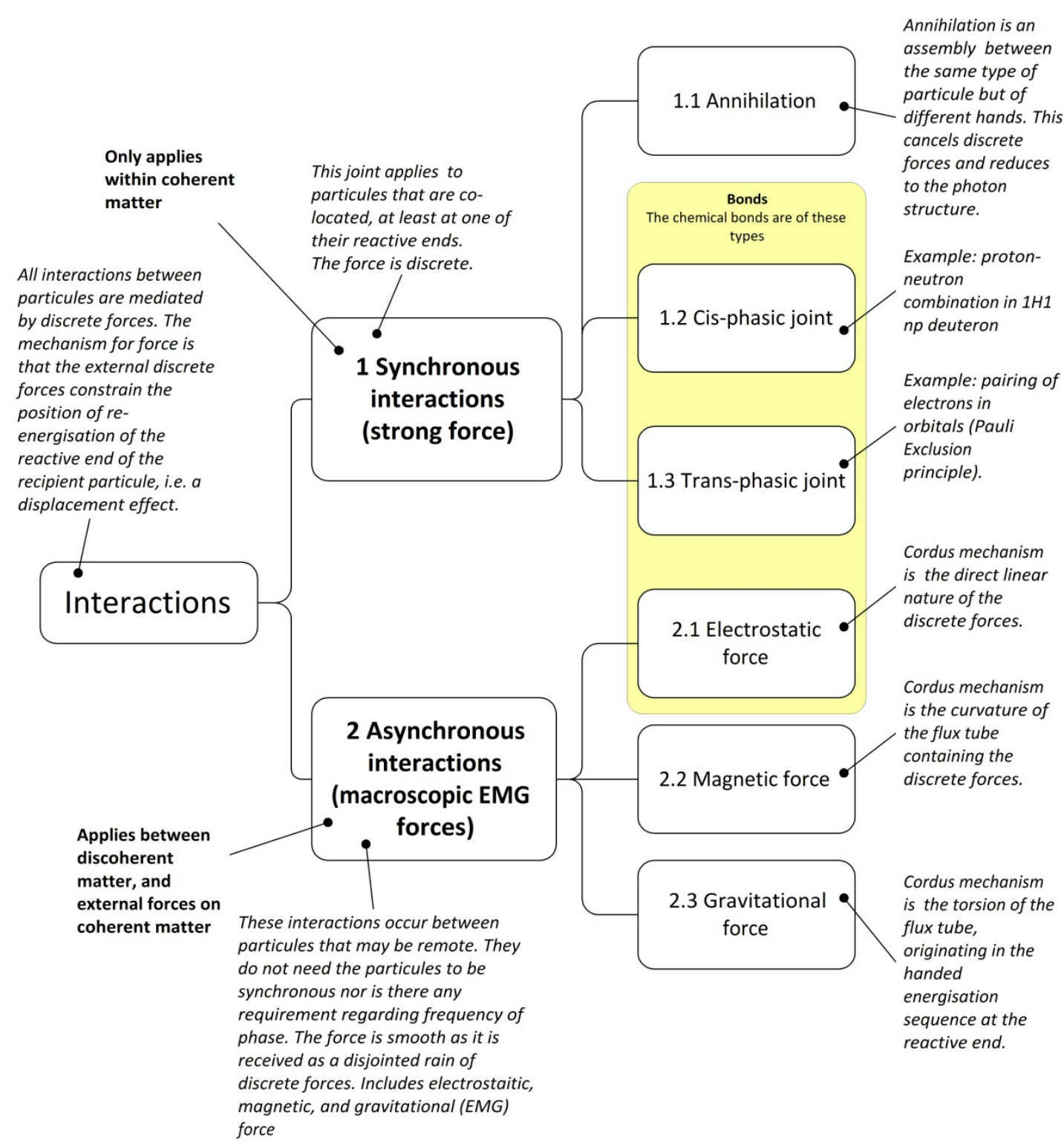

Figure 7. Cordus force hierarchy theory. All interactions between particules are mediated by discrete forces. The mechanism for force is that the discrete forces constrain the position of re-energisation of the reactive end of the recipient particule, i.e. a displacement effect. The state of the particules, particularly the synchronicity and phase of their frequency, results in several types of forces/interactions as shown

Thus the Cordus theory proposes a single mechanism, the discrete forces, for all the forces. The forms of the strong force that particularly interest us here are the cis- and trans-phasic forms, because these are implicated as determinants of the nuclear structure. This Cordus theory also proposes that the term 'strong' force (interaction) is a misnomer. 'Synchronous' interaction is a better description of the mechanisms, if we are correct.

\section{Discussion}

\subsection{What Has Been Achieved?}

\section{Overview}

Using the Cordus conjecture we make several new proposals regarding the forces (interactions). Specific subcomponents to this theory are:

1) Mechanisms are proposed for the strong force, in terms of the interlocking of discrete field elements (discrete forces) between particules. This is a novel alternative perspective. 
2) A single mechanism, the discrete force, is proposed to underlie all of the strong force, annihilation, and the EMG forces. This is also a novel alternative perspective.

3 ) The strong force is predicted to be intimately linked to coherent assemblies of matter. The theory predicts the EMG forces are the associated discoherent phenomenon. This also means that any one particule will influence another either via the strong force or the EMG forces, not both. Consequently there is no need to switch off or overcome the electrostatic force, hence we suggest that 'strong' is an incorrect way of thinking about this interaction. This is another novel alternative perspective.

\section{Reconceptualisation of the strong force}

This paper makes the contribution of providing a new mechanism for the strong interaction. The attributes conventionally expected of the nuclear force are: that it is attractive between nucleons whether neutral neutrons or positively charged protons; that it is repulsive at close range; that its effect drops off with range. The Cordus theory accommodates all these, though in radically different ways. Thus in Cordus the nuclear force is caused by the alignment of flux lines (HEDs) between bonding particules, and the interlocking of the discrete forces in those lines. Those discrete forces are renewed at the de Broglie frequency of the particule. Thus we propose the equivalence of mass, energy, frequency, and the inverse of the span, i.e. an extension of the Plank relation, at the particule level. We also provide a physically meaningful interpretation of the de Broglie frequency, which is otherwise an abstract variable.

Conceptually what we have achieved, using a design method, is to transform the inefficient conventional requirements for the strong force (weak $>$ strongly attractive $>$ repulsive) into another solution space where it can be represented with a much simpler and more efficient solution. Doing so also integrates the electrostatic force into the solution, which is another advantageous feature of the design.

The Cordus theory predicts that the strong force will be unable to bond all types of particules: those that cannot obtain a frequency match (or a harmonic thereof) will not be bonded this way but will feel the EMG forces instead. This may be testable and falsifiable.

Thus the Cordus theory also readily explains why the nuclear force depends on the alignment of the spins of the nucleons (a known effect). The explanation is that one reactive end of the first particule is energising and its other is de-energising, and the correct end needs to aligned with the second particule, hence an orientation or 'spin'. The QM concept of spin becomes, in the Cordus theory, the angular orientation of the fibril (or phase angle) of the particule. But providing the spin criterion is met then the interaction is simply based on phased interlocking of discrete forces, so the force can bind particles where electrostatic forces would otherwise not allow.

The interlocking of the discrete forces requires the participants to have the same frequency (or a harmonic) and thus we predict that the interaction also couples the energy systems of the particules, and hence also their geometric spans. The synchronous interaction (strong force) is therefore, according to the Cordus interpretation, a constraint on the location of re-energisation of a reactive end. Consequently the interlocking also prevents the particules from moving away from or closer to each other. Thus the attractive and repulsive features of the nuclear force are accommodated: we do not need a separate mechanism to prevent the strong force from crushing the assembly into a singularity.

As the reactive end of a particule tries to move out of a nuclear relationship, it encounters synchronisation constraints that pull it back into interlock. This also explains the known effect whereby a particule that gains sufficient energy may break out of the relationship. Likewise decay is explained in the Cordus theory as particules that were not very strongly bound together in the first place, being vulnerable to obtaining sufficient energy, e.g. from perturbation by discrete forces in the fabric, to escape the relationship. Also, in the Cordus theory there is no singularity when one reactive end from each particule co-locates, and hence collapse is not an issue.

\section{Provision of a conceptual framework for unification of forces}

A second contribution is the provision of a novel holistic framework for the unification of forces. This theory encompasses the strong and electro-magneto-gravitational forces. They all rely on discrete forces. The EMG forces are proposed to be based on the linear strength, bending, and torsional deflection (respectively) caused by these discrete forces and their flux lines (Cordus: hyff), whereas the strong force is based on the synchronicity of the discrete forces. The theory provides a qualitative explanation of the mechanism for transmission of those forces and the fundamental mechanism of force (prescribed displacement of reactive ends). This unification is achieved by the discrete forces being common to all the interactions. The weak force is not a force at all, 
according to the Cordus theory, but rather a decay process for neutrons, and has different proposed causality.

\section{Integrating coherence into the force theory}

A third contribution is the conceptual integration of coherence into the force theories. Several novel propositions emerge from this line of thinking, e.g. the idea that the strong force is simply another aspect of coherence within matter, whereas the EMG forces are felt when matter is discoherent, see Implications below. Likewise, that annihilation is a type of strong-force interaction.

Crucially, these concepts only become conceivable for a Cordus type particule with its additional dimensions (internal variables). From an epistemic perspective, what has happened here is an expansion of the dimensions available to a particule. In particular, QM constructs particles as merely zero-dimensional points, and therefore cannot conceive of anything but a single strong force. Concepts like 'spin' and 'frequency' are only abstract concepts to QM: it cannot give them any meaning. Consequently QM relies on creating more particles whenever it needs more variables to explain something, as evident in its creation of virtual gauge bosons and the gluons. In contrast, the Cordus theory provides particules with several additional dimensions: span, frequency, phase, and orientation angles, and is therefore more parsimonious in its use of variables. (Note 5)

\subsection{Comparison with $Q C D$}

Quantum chromodynamics provides a theory of this interaction at the level of quarks, based on colour-charge. QCD models the force as occurring by the quarks transmitting and exchanging gluons with each other. QCD requires multiple forces and messenger particules. There is an electrostatic force (transmitted by virtual photons) that stays on at all scales. This must then be overcome by the nuclear force (transmitted by virtual pions) which in turn is a derivative of the colour force (transmitted by gluons). It is not yet possible to model nuclear structure from the ground up using QCD.

The Cordus theory provides a different construct: it proposes that a particular behaviour of the discrete forces carries the strong force. Where QCD has gluons, the Cordus theory has discrete forces, though they are not equivalent. The Cordus explanation is that QCD's colour charge (a charge-like property of quarks and gluons) corresponds to the three hyff emission directions (HEDs) and the discrete forces therein. So the outcomes are similar, but the mechanisms and conceptual foundations are altogether different. The Cordus theory has a much wider role for its discrete forces than QCD has for its gluons. We propose that the discrete forces determine both the strong and nuclear force, make up the wider electro-magnetic-gravitational (EMG) fields, provide a discrete force field, and make up the fabric of space and time (D. J. Pons et al., 2013). Thus we propose that the electrostatic force (and EMG forces in general) is merely the discrete force at the larger scale where synchronisation no long applies. So the strong force is writ large on the universe.

In the Cordus theory there is a single conceptual theory for both the strong interaction between quarks and the nuclear force between nucleons. The pions are readily accommodated in the Cordus matter-antimatter theory, but are not needed to explain the nuclear force. So there need be only one force operating at the nucleon-to-nucleon interaction, not multiple. No new particles are required. No separate mechanism is necessary for repulsion, because in this theory the repulsion is not a separate force but a consequence of the strong central tendency of the synchronous interaction.

The Cordus theory also obviates the need for the families of 'virtual particles' of the standard model: neither virtual photon nor graviton, nor any other force boson is required. It is not that these are disallowed. Rather the Cordus theory permits that transients in the vacuum fabric of background discrete forces may be interpreted as conventional virtual point-particles. It is just that the Cordus theory shows that a much richer, and also more ontologically efficient, interpretation is available by abandoning the point perspective.

The Cordus synchronous theory suggests: (a) that QM is a mechanics only for the special case of coherent assemblies of particles, i.e. the regime of the synchronous interaction, and is not applicable to discoherent assemblies, and (b) that even in the case of coherent assemblies, QM is a simplification of a deeper determinism. The source of that simplification is QM's dependence on the 0-D point premise.

\subsection{Implications for Coherent Bodies}

So we have provided a radical reconceptualisation of the strong force. If this is a valid solution, then it has some interesting implications as follow.

Conventionally the strong force overcomes the electrostatic repulsion of protons. But the Cordus theory proposes a totally different mechanism whereby one particule affects another either by the strong force, or the EMG forces, not both. 
Applying this to the nucleus, this implies that immediate neighbouring nucleons would interact by the strong force, and potentially feel the effect of more distant nucleons (in the same atom or other atoms) as the EMG forces (particularly the electrostatic since this is a direct tension/compression whereas the magnetic requires relative motion, and the gravitational is weak). This is consistent with the liquid drop model of the nucleus.

In the nucleus the arrangement of nucleons will possibly, even likely, be geometrically complex, such that nucleons far from each other in the assembly may perceive the EMG forces (primarily electrostatic) rather than the strong force. One could alternatively say that the coherence is short-ranged. Certainly at the level of molecules the interaction is generally understood to be electrostatic rather than the strong force. However there is the possibility that macroscopic coherent bodies, e.g. superfluids, may have the strong force synchronised throughout the whole body. Below we explore the intriguing possibilities and novel predictions if this were to be the case.

\section{Alternative explanation for superconduction effects}

We have previously given an explanation for the expulsion of magnetic fields from superconductors (the Meissner effect), in terms of the coherent complementary frequency synchronisation (CoFS) network providing lateral stiffness: the discrete forces from neighbouring electrons lock the nodes of the entire network in place (D. J. Pons, A. D. Pons, A. M. Pons, \& A. J. Pons, 2011b). Therefore an external magnetic field cannot displace the reactive ends: its effect is resisted, and the flux lines are denied passage so they go round the wire instead. Surface currents arise as compensatory consequences of the load on the synchronised network of electrons. We now suggest another explanation, complementary to the first: that a coherent body (of electrons in the case of a superconductor) uses the strong force to support that coherence and therefore cannot use it to also create EMG forces within the body. Thus a second, complementary, explanation is provided for the Meissner effect.

\section{Implications for response of a coherent body to EMG fields}

This theory suggests that all EMG forces between neighbouring bonded coherent particules are switched off because the interaction manifests as the strong force instead. There are some curious implications for gravitation. If, as the Cordus sub-theory for force suggests, the EMG forces are linear, bending, and torsional effects respectively of the hyff, and given that Cordus explains coherence as a regime of the strong interaction that applies step-wise throughout a body, then the logic implies that a coherent body may also feel only the strong force (not the self-EMG forces) internally.

Taken together, we can thus make some tentative predictions for a coherent body, e.g. a superconductor, superfluid or supersolid, and these may be falsifiable:

1) This Cordus theory predicts that electric and magnetic forces, and presumably also gravitational, cannot be transmitted within a coherent body. The coherent body as a whole is predicted to respond to external EMG forces at its skin layer. Thus coherent bodies should be unable to sustain an internal voltage gradient, magnetic field, or gravitational field. This should apply to superfluids too. The prediction regarding gravitation is likely to be controversial, as gravity is otherwise thought to be unshieldable. This is falsifiable.

2) The EMG fields emitted outwards by a coherent body are predicted to be pulsed (like a laser) and not continuous (as opposed to discoherent bodies emitting macroscopically smooth fields).

a. The frequency will be that of the basic individual particule, not the mass of the body as a whole.

b. The frequency will be independent of the number of particules in coherence.

c. The fields will be polarised.

3) The categorisation into EMG vs. strong force leads us to predict that self-gravitation (i.e. of internal origin) too is inoperative within a coherent body.

d. Specifically, that self-gravity does not operate in coherent bodies: the atoms are not gravitationally attracted to each other. Thus such a body should not collapse under self-weight (at least while the coherence is able to be maintained).

e. High gravitational loading, presumably acceleration too, may be able to overload the skin and cause disccoherence of the body as a whole.

f. We anticipate that external gravitational fields should interact with the coherent body at its skin, not with its bulk.

g. External gravitational fields are presumably also diverted around a coherent body.

This is a peculiar combination of unusual predictions, and could be testable. 


\subsection{Limitations}

The ideas expressed here are founded in a radically different concept for 'particle', and cut across conventional thinking. This is a thought experiment which starts with the question, 'What if particles were not zero-dimensional points?' We have shown that this is a valid question to ask, the Bell-type inequalities notwithstanding. It is also an interesting question to ask, and surprising answers are possible. Thus we make no apology for the unorthodoxy of the concepts expressed here, though we are likewise quick to point out that the conjectural nature of the Cordus theory is naturally a limitation. The theory has good fitness to qualitatively explain a diverse variety of phenomena, but this is still not validation. At this stage it is primarily a candidate conceptual theory. Being primarily a conceptual work, there is not yet any published mathematical formalism of the theory: we identify this as an opportunity for future development.

\subsection{Opportunities for Further Research}

NLHV designs suggest many opportunities for further empirical research and breakthrough conceptual development. The ultimate research question would be to explain nuclear structure from the strong force upwards. An ideal nuclear theory would explain the range of nuclides observed, predict why some are more or less stable than others, explain why certain nuclides are uncharacteristically unstable (e.g. ${ }_{4} \mathrm{Be}_{4}$ ), and explain the trends in the progression of the stable/unstable nuclei. Obviously this is beyond the capability of all existing nuclear theories, and is an area where the NLHV sector has the potential to make interesting new contributions.

\section{Conclusions}

The purpose of this work was to create a new conceptual theory of the strong force. We have achieved that, and in the process provided a novel explanation for the other forces too.

\section{A new conceptual theory of the synchronous interaction (strong force) has been developed, including a qualitative description of the proposed mechanics.}

The central idea is based on the Cordus particule structure and predicts that the strong interaction arises from the synchronisation of discrete forces. This causes the reactive ends of participating particules to be interlocked: the interaction pulls or repels particules into co-location and then holds them there. This readily explains the attractive-repulsive nature of that force, and short range. It is predicted that this force only applies to particules in coherent assembly.

\section{A new conceptual theory for the unification of the strong and electro-magneto-gravitation (EMG) forces has been provided.}

The Cordus theory proposes that the EMG forces and the strong force are different manifestations of a single underlying mechanism. The EMG forces are proposed to be based on the linear strength, bending, and torsional deflection (respectively) of the flux lines (Cordus: hyff) of these discrete forces, whereas the strong force is based on the synchronicity of the discrete forces.

In addition this theory makes the novel prediction that the nature of the force perceived by a particule depends on whether or not it is in a coherent relationship with the emitting particule. The synchronous interaction (strong force) is the more fundamental interaction: it binds the particules together by creating interlocked constraints on the geometric location of the particules, if they are coherent. The discrete forces then propagates outwards, exerting the EMG forces on other more remote particules that it encounters.

The important implication of this theory is that any one particule can perceive the fields of another external particule EITHER as the strong force, OR as EMG forces, not both. Which it is depends on whether or not they are coherent (respectively). Thus the nature of their bonding and their proximity determines the forces they receive. Thus also the strong force is predicted to be intimately linked to coherence, with the EMG forces being the associated discoherent phenomenon. All these forces are simply manifestations of a governing synchronous interaction at the fundamental level.

These concepts only make sense in the context of a particule with an internal structure, and are inaccessible to the 0 -D point construct of the standard model of physics. Thus we have also shown that it is worthwhile questioning the fundamental premises of physics, for the interesting outcomes that can result.

We therefore challenge the conventional construct of the strong force, namely its weak $>$ attractive $>$ repulsive nature. We show it is possible to conceive of a single deeper mechanism, based on synchronicity, that displays all these behaviours without needing multiple separate mechanisms. Doing this not only solves the strong force problem more effectively and parsimoniously than QCD, but also unifies the forces (except the weak interaction which we contend is not a force). 


\section{References}

Bell, J. S. (1964). On the Einstein podolsky rosen paradox. Physics, 1(3), 195-200.

De Zela, F. (2008). A non-local hidden-variable model that violates Leggett-type inequalities. Journal of Physics A: Mathematical and Theoretical, 41(50), 505301. http://dx.doi.org/10.1088/1751-8113/41/50/505301

Gamow, G. (1930). Mass defect curve and nuclear constitution. Proceedings of the Royal Society of London, 126, 632-644. http://dx.doi.org/10.1098/rspa.1930.0032

Gell-Mann, M. (1962). Symmetries of Baryons and Mesons. Physical Review, 125(3), 1067-1084. http://dx.doi.org/10.1103/PhysRev.125.1067

Groblacher, S., Paterek, T., Kaltenbaek, R., Brukner, C., Zukowski, M., Aspelmeyer, M., \& Zeilinger, A. (2007). An experimental test of non-local realism. Nature, 446(7138), 871-875. http://dx.doi.org/10.1038/nature05677

Ivanenko, D. (1985). Remarks on the establishing the theory of nuclear forces. Progress of Theoretical Physics, 85, 20-26. http://dx.doi.org/10.1143/PTPS.85.20

Laudisa, F. (2008). Non-Local realistic theories and the scope of the bell theorem. Foundations of Physics, 38(12), 1110-1132. http://dx.doi.org/10.1007/s10701-008-9255-8

Leggett, A. (2003). Nonlocal hidden-variable theories and quantum mechanics: An incompatibility theorem. Foundations of Physics, 33(10), 1469-1493. http://dx.doi.org/10.1023/a:1026096313729

Nambu, Y., \& Han, M. Y. (1974). 3 Triplets, paraquarks, and colored quarks. Physical Review D, 10(2), 674-683. http://dx.doi.org/10.1103/PhysRevD.10.674

Pons, D. J. (2011). Stability and decay: Mechanisms for stability and initiators of decay in the neutron. Vixra, 1112.0002, 1-17. Retrieved from http://vixra.org/pdf/1112.0002v1.pdf

Pons, D. J. (2012). Limits of Coherence: Where and why is the transition to discoherence? Vixra, 1201.0043, 1-12. Retrieved from http://vixra.org/abs/1201.0043

Pons, D. J., Pons, A. D., \& Pons, A. J. (2011). The preponderance of matter: Asymmetrical genesis via the antineutrino route. Vixra, 1111.0035, 1-19. Retrieved from http://vixra.org/pdf/1111.0035v1.pdf

Pons, D. J., Pons, A. D., \& Pons, A. J. (2012). Bundles of nothingness: unravelling the zero-dimensional particle premise of fundamental physics. Foundational Questions Institute: Essay Contest 2012: Questioning the Foundations, 1-12. http://fqxi.org/community/forum/topic/1334

Pons, D. J., Pons, A. D., \& Pons, A. J. (2013a). Differentiation of matter and antimatter by hand: internal and external structures of the electron and antielectron. Vixra, 1305.0157, 1-18. Retrieved from http://vixra.org/pdf/1305.0157v2.pdf

Pons, D. J., Pons, A. D., \& Pons, A. J. (2013b). Time: An emergent property of matter. Vixra, 1301.0074, 1-42. Retrieved from http://vixra.org/abs/1301.0074

Pons, D. J., Pons, A. D., Pons, A. M., \& Pons, A. J. (2011a). Matter particuloids. (Cordus matter Part 3.2) Vixra, 1104.0023, 1-12. Retrieved from http://vixra.org/pdf/1104.0023v1.pdf

Pons, D. J., Pons, A. D., Pons, A. M., \& Pons, A. J. (2011b). Special states of matter. (Cordus matter Part 3.4) Vixra, 1104.0025, 1-12. Retrieved from http://vixra.org/pdf/1104.0025v1.pdf

Pons, D. J., Pons, A. D., Pons, A. M., \& Pons, A. J. (2012). Wave-particle duality: A conceptual solution from the $\begin{array}{lllll}\text { cordus } & \text { conjecture. } & \text { Physics }\end{array}$ http://physicsessays.org/doi/abs/10.4006/0836-1398-25.1.132

Weizsäcker, C. F. V. (1935). Zur Theorie der Kernmassen (The theory of nuclear mass). Zeitschrift für Physik, 96 , 431-458. http://dx.doi.org/10.1007/BF01337700

Wilczek, F. (2005). Asymptotic freedom: From paradox to paradigm. Proceedings of the National Academy of Sciences, 102(24), 8403-8413. http://dx.doi.org/10.1073/pnas.0501642102

Yukawa, H. (1947). On the theory of elementary particles .1. Progress of Theoretical Physics, 2(4), 209-215. http://dx.doi.org/10.1143/PTP.2.209 


\section{Notes}

Note 1. Within the theory these discrete force pulses are termed as vires. The terms vis (singular) and vires (plural) are Latin for force. We acknowledge that we have not described what comprises these discrete forces, or the flux lines. Instead, the Cordus conjecture simply shows that having such elements is a logical necessity for this solution.

Note 2. The proton has two discrete forces in one of the axes, hence the designation $\mathrm{p}\left(\mathrm{r}^{1}{ }_{1.1} \cdot \mathrm{a}_{1}\right.$. $\left.\mathrm{t}_{1}\right)$. Note the use of $r_{1.1}^{1}$ as opposed to say $r_{2}^{1}$. This is because the theory does not require that the two discrete forces are emitted simultaneously. We therefore do not need to unnecessarily limit the thinking in this area, as $\mathrm{r}_{2}^{1}$ might imply. We hypothesise that the particule needs to service this finer internal structure, and slices its frequency accordingly. The corollary is that the phenomenon may also explain rest mass and generations.

Note 3. The Cordus explanation for force is that the discrete forces apply a prescribed constraint on the location, hence displacement, of the reactive end that the particule that receives them. This is conceptualised as the incoming discrete forces interfering with the emission of the particule's own discrete forces through an energy coupling, hence a change in frequency, hence also a change in span of the particule, and thus a change in position of the reactive ends: an effect that is observed as a tendency to displacement or strain. In the application of this to the synchronous interaction, it is important to remember that this interaction requires an assembly and frequency interlock of one reactive end from each of two or more different adjacent particules, as opposed to the EMG interactions where the particules are ranged and desynchronised regarding frequency. This also means that the synchronous interaction avoids the singularity problem that troubles other nuclear theories: the assembly of Cordus particules takes up space according to the length of the spans of the participating particules. As Cordus span is inversely related to frequency, and accepting the frequency, energy and mass equivalence, this theory also explains why high energy (heavier) particules are smaller.

Note 4. For Cordus particules assembled with the synchronous interaction, it is essential that they redistribute energy between them. This is because any energy difference in one matter particule will cause its frequency, and hence also the span, to change. In turn both these changes will put pressure on the particule to desynchronise. The HED-based strong force resists that, and the discrete forces transfer the excess energy to the other particule. If the input energy is too great for the HED negotiation to accommodate, then the particules disassemble.

Note 5. The design methodology, by which the Cordus theory was developed, simply identifies the number and type of variables necessary and sufficient to explain the fundamental phenomena under examination. If one wishes to consider each Cordus internal variable a dimension, then the tally is three linear dimensions [x, $y, z]$ for location of a reactive end, one for the length of the span (related to energy of the particule), three polarisation angles for the orientations of the HEDs (field emission directions) assuming that the [r] axis is not necessarily always in the linear direction of the span (which we think is the case with the photon though we are unsure about massy particules), one composite variable for each of three HEDs to denote the field activation status (number and directions of discrete forces) of that HED, and one variable to indicate which reactive end is energising (spin). Not all these dimensions are ratio variables. That gives a total of 11 (or 10) variables (dimensions) to fully define a Cordus particule. This is similar to some versions of $\mathrm{M} /$ string theory. Note that time is not included: the Cordus theory does not accept macroscopic time as another dimension, as is the common interpretation of conventional physics.

\section{Copyrights}

Copyright for this article is retained by the author(s), with first publication rights granted to the journal.

This is an open-access article distributed under the terms and conditions of the Creative Commons Attribution license (http://creativecommons.org/licenses/by/3.0/). 